\title{
Dynamical structure underlying inverse stochastic resonance and its implications
}

\author{
Muhammet Uzuntarla, ${ }^{1, *}$ John R. Cressman, ${ }^{2, \dagger}$ Mahmut Ozer, ${ }^{3}$ and Ernest Barreto ${ }^{2, \ddagger}$ \\ ${ }^{1}$ Department of Biomedical Engineering, Bulent Ecevit University, Zonguldak, Turkey \\ ${ }^{2}$ Department of Physics, Astronomy, and Computational Sciences and The Krasnow Institute for Advanced Study, \\ George Mason University, Fairfax, Virginia, USA \\ ${ }^{3}$ Department of Electrical-Electronics Engineering, Bulent Ecevit University, Zonguldak, Turkey
}

(Received 25 March 2013; published 31 October 2013)

\begin{abstract}
We investigate inverse stochastic resonance (ISR), a recently reported phenomenon in which the spiking activity of a Hodgkin-Huxley model neuron subject to external noise exhibits a pronounced minimum as the noise intensity increases. We clarify the mechanism that underlies ISR and show that its most surprising features are a consequence of the dynamical structure of the model. Furthermore, we show that the ISR effect depends strongly on the procedures used to measure it. Our results are important for the experimentalist who seeks to observe the ISR phenomenon.
\end{abstract}

DOI: 10.1103/PhysRevE.88.042712

PACS number(s): 87.19.1c, 05.40.-a, 05.45.-a

\section{INTRODUCTION}

The spiking of a neuron can be thought of as a stochastic process due to the presence of various sources of noise [1]. Stochasticity is manifested most obviously by the highly variable spike train patterns of neurons in vivo produced by a repeated sensory input $[2,3]$ and by the spontaneous neuronal activity in the absence of any stimuli [4,5]. Noise can also enrich the stochastic dynamics of neuronal ensembles and facilitate the information processing capabilities of neurons. For example, stochastic resonance (SR) is a well-known phenomenon by which the weak-signal processing ability of neurons can be enhanced [6-9]. Previous experimental and theoretical studies have suggested that noise can improve the detection, integration, and transmission efficiency of signals, and induces many complex behaviors such as synchronization and burst firing [10-13].

On the other hand, some recent works have been concerned with the inhibitory effects of noise on neuronal activity, particularly on rhythmic firing. Paydarfar et al. [14] studied the influence of noise on neuronal pacemakers in an in vitro preparation of squid axon and found that small noisy currents can induce on-off switching behavior between repetitive firing and quiescence. They also showed that the timings of the switching depend on the intensity and spectral properties of the noisy current. Gutkin, Tuckwell, and Jost $[15,16]$ investigated an inhibitory effect of noise in a single Hodgkin-Huxley model neuron. They reported that for a range of mean input current densities near the critical value that leads to rhythmic firing, there exists a minimum —or even silence-in spiking activity for a moderate range of noise intensities. (Earlier work by the same authors considered a similar effect in a pair of

\footnotetext{
*muzuntarla@yahoo.com

†jcressma@gmu.edu

†ebarreto@gmu.edu
}

Published by the American Physical Society under the terms of the Creative Commons Attribution 3.0 License. Further distribution of this work must maintain attribution to the author(s) and the published article's title, journal citation, and DOI. reciprocally coupled model neurons [17].) By analogy with stochastic resonance, the authors called this noise-dependent minimum in firing rate "inverse stochastic resonance" (ISR). Recently, Guo [18] investigated the impact of noise structure on ISR and found that the inhibitory effect of colored noise is stronger than that of the Gaussian white noise studied in Refs. [15,16]. Tuckwell and Jost [19] extended the subject to a spatially extended Hodgkin-Huxley system and showed that if the noise and signal (mean current) are uniformly distributed along the whole spatial extent of the neuron, weak noise could strongly inhibit the occurrence of rhythmic spiking, but not its propagation. However, when the noise and signal are applied to separate regions of the neuron, the noise has no effect on either rhythmic spiking or the propagation of spikes.

In these modeling studies [14-16,18,19], noise was incorporated by adding an external additive noisy current. A more realistic biophysical approach is to consider the channel noise that results from the stochastic dynamics of voltage-gated ion channels, i.e., the random transitions between open and closed states. (See also Ref. [20], where noise from synaptic background activity with unreliable synapses was considered.) Much work has been devoted to understanding the role of channel noise on neuronal dynamics, and it has been shown that channel noise can modify excitability, cause spontaneous firing, induce synchronization in neural networks, improve performance of SR, and result in variability of spike timing as well as interspike intervals [21-25].

Here, we consider the ISR phenomenon in the HodgkinHuxley neuron using this approach. We explicitly model noise as resulting from the stochastic nature of voltage-gated ion channels embedded in the neuronal membrane. We assume a fixed channel density so that the intensity of channel noise is inversely related to membrane area. Our results show that the ISR phenomenon is also present in this more biophysical case. However, we will argue below that any reasonable model of noise can be used to elicit the ISR phenomenon.

Our main contribution in this work is to analyze and clarify the mechanism that underlies ISR from a nonlinear dynamics perspective. We show that the most surprising feature-the increase in average firing rate as noise decreases (or membrane area increases) -is a consequence of both the dynamical structure of the model and the particular measuring 
procedures used. This means that different ways of measuring the ISR effect yield quantitatively and potentially qualitatively different results. We demonstrate this explicitly and confirm our interpretation with a simple theoretical calculation.

\section{MODEL AND METHODS}

The original Hodgkin-Huxley model describes the time evolution of the transmembrane potential in terms of membrane currents as follows [26]:

$$
\begin{aligned}
C_{m} \frac{d V}{d t}= & -G_{\mathrm{Na}}(m, h)\left(V-E_{\mathrm{Na}}\right)-G_{K}(n)\left(V-E_{K}\right) \\
& -G_{L}\left(V-E_{L}\right)+I_{0},
\end{aligned}
$$

where $V$ represents the membrane potential in millivolts, and $C_{m}=1 \mu \mathrm{F} / \mathrm{cm}^{2}$ is the membrane capacitance per unit area. $I_{0}$ is a constant external current stimulus in $\mu \mathrm{A} / \mathrm{cm}^{2}$ and is used for the modulation of neuronal excitability. Here, since we assume that the source of noise is primarily internal, $I_{0}$ represents either an injected current or a constant average synaptic input to the neuron. $G_{\mathrm{Na}}, G_{K}$, and $G_{L}$ denote sodium, potassium, and leak conductances, respectively. The parameters $E_{\mathrm{Na}}=115 \mathrm{mV}, E_{K}=-12 \mathrm{mV}$, and $E_{L}=10.6 \mathrm{mV}$ are the corresponding reversal potentials. In the model, the leakage conductance is assumed to be constant, with $G_{L}=$ $0.3 \mathrm{mS} / \mathrm{cm}^{2}$, while the sodium and potassium conductances change dynamically according to the following equations:

$$
\begin{gathered}
G_{\mathrm{Na}}(m, h)=g_{\mathrm{Na}}^{\max } m^{3} h \\
G_{\mathrm{K}}(n)=g_{\mathrm{K}}^{\max } n^{4},
\end{gathered}
$$

where $g_{\mathrm{Na}}^{\max }=120 \mathrm{mS} / \mathrm{cm}^{2}$ and $g_{\mathrm{K}}^{\max }=36 \mathrm{mS} / \mathrm{cm}^{2}$ are the maximal sodium and potassium conductances, respectively. Moreover, $m$ and $h$ stand for the activation and inactivation gating variables for the sodium channel, respectively, and the potassium channel includes an activation gating variable $n$.

To incorporate channel noise into the HH model, different computational algorithms have been proposed by various groups [22-24,27]. In the present study, we use the well-known algorithm of Fox [27], because it is widely used and is more computationally efficient than other algorithms. According to this approach, the gating variables obey the following Langevin generalization:

$$
\frac{d x}{d t}=\alpha_{x}(1-x)-\beta_{x} x+\xi_{x}(t)
$$

where $\alpha_{x}$ and $\beta_{x}$ (with $x=m, n, h$ ) are the experimentally determined voltage-dependent rate functions for the gating variable $x$ and can be found in Refs. [26,27]. The stochastic nature of the channels appear via the independent Gaussian noise sources $\xi_{x}(t)$ in Eq. (4) with $\left\langle\xi_{x}(t)\right\rangle=0$ and $\left\langle\xi_{x}(t) \xi_{x}\left(t^{\prime}\right)\right\rangle=D_{x} \delta\left(t-t^{\prime}\right)$, for $x=m, n, h$. The $D_{x}$ denote the intensities of the various channel noises, which are inversely proportional to the total number of channels of each type in a membrane patch of area $A$, as follows [27]:

$$
\begin{aligned}
D_{m} & =\frac{2}{N_{\mathrm{Na}}} \frac{\alpha_{m} \beta_{m}}{\alpha_{m}+\beta_{m}} \\
D_{h} & =\frac{2}{N_{\mathrm{Na}}} \frac{\alpha_{h} \beta_{h}}{\alpha_{h}+\beta_{h}}
\end{aligned}
$$

$$
D_{n}=\frac{2}{N_{K}} \frac{\alpha_{n} \beta_{n}}{\alpha_{n}+\beta_{n}},
$$

where $N_{\mathrm{Na}}$ and $N_{K}$ represent the total number of the sodium and potassium channels within the membrane patch. Assuming homogenous sodium and potassium ion channel densities $\rho_{\mathrm{Na}}=60 \mu \mathrm{m}^{-2}$ and $\rho_{K}=18 \mu \mathrm{m}^{-2}$, the total number of channels is $N_{\mathrm{Na}}=A \rho_{\mathrm{Na}}$ and $N_{K}=A \rho_{K}$ [27-30]. Equations (1)(7) constitute the stochastic Hodgkin-Huxley model, where the membrane area $A$ globally determines the intrinsic noise level.

Numerical integration of our system was carried out using the standard Euler algorithm with a step size of $10 \mu \mathrm{s}$.

\section{RESULTS}

We begin by showing that our channel-noise model reproduces the previously reported ISR phenomenon. Following the procedure in Ref. [16], we calculate the average firing rate of our model neuron for fixed values of membrane area $A$ and input current $I_{0}$ as follows. First, an initial condition is randomly selected with uniform probability within a fixed region of the four-dimensional state space $(V, m, n$, and $h)$, which we call the state space sample volume. Specifically, this region ranges from -10 to $80 \mathrm{mV}$ for the transmembrane voltage variable $V$, and from 0 to 1 for the each of the gating variables $m, n$, and $h$. Then, the system [Eqs. (1)-(7)] is integrated for a time $T$. After this, we count the number of spikes $N_{\text {spikes }}$ that occur in an additional time interval of length $\tau$. Each spiking event is defined by the upward crossing of the membrane potential past a threshold of $20 \mathrm{mV}$. This entire procedure is repeated $N$ times, and an average spiking rate is calculated as

$$
r\left(A, I_{0}\right)=\frac{1}{N \tau}\left(\sum_{i=1}^{N} N_{\text {spikes }, i}\right),
$$

where the index $i$ refers to each trial.

In Fig. 1(a), $r$ is plotted versus the membrane area $A$ for $I_{0}=6.8 \mu \mathrm{A} / \mathrm{cm}^{2}, T=1 \mathrm{~s}, \tau=5 \mathrm{~s}$, and $N=1000$. Since the membrane area is inversely proportional to the channel noise intensity, weak noise is to the right, and the noise intensity increases toward the left. Note that the average firing rate is lowest for moderate values of the noise intensity. This is the ISR phenomenon.

The error bars represent the standard deviation at each point. Figures 1(b) and 1(c) show histograms of $N_{\text {spikes }}$ for $A=750 \mu \mathrm{m}^{2}$ and $30000 \mu \mathrm{m}^{2}$, respectively. These values correspond to the tick marks on the horizontal axis in Fig. 1(a). The starkly bimodal distribution shown in Fig. 1(c) is representative of the distributions for all the corresponding data points for which $A \geqslant 1500 \mu \mathrm{m}^{2}$, except where the average is small. In those cases, the peak at zero dominates.

To understand this phenomenon, we examine the nature of the solutions during the time intervals in which the spikes are counted. Several voltage traces for $I_{0}=6.8 \mu \mathrm{A} / \mathrm{cm}^{2}$ and $A=750 \mu \mathrm{m}^{2}$ are shown in Fig. 2(a). Each trace originates from a randomly chosen initial condition as described above and shows the neuron's behavior after the transient time $T$ has passed. Many random noise-induced transitions from resting to spiking and vice versa can be seen. Accordingly, 

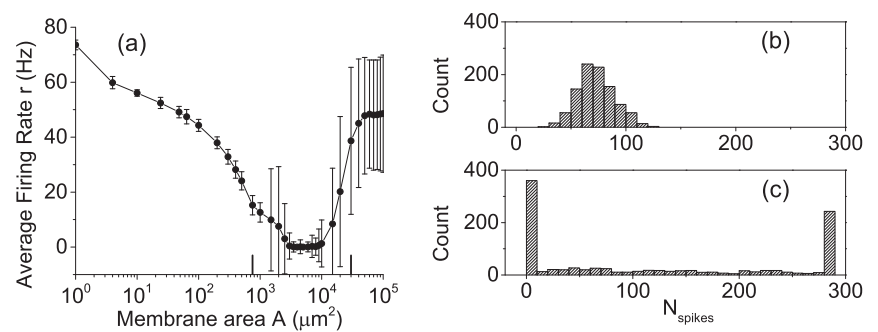

FIG. 1. (a) Inverse stochastic resonance. The average firing rate $r$ is plotted versus the membrane area $A$ for $I_{0}=6.8 \mu \mathrm{A} / \mathrm{cm}^{2}$. For each value of the membrane area, $N=1000$ trajectories starting from randomly chosen initial conditions were obtained as described in the main text. The average firing rate was calculated based on the number $N_{\text {spikes }}$ of spikes that occur in a $\tau=5$-s window, after discarding the initial $T=1$-s transient. The error bars represent the standard deviation. Since the noise intensity is inversely proportional to the membrane area, weak noise is to the right (large membrane area), and stronger noise is to the left (small membrane area). The dip in the curve suggests an inverse resonance. (b), (c) Histograms of $N_{\text {spikes }}$ corresponding to $A=750 \mu \mathrm{m}^{2}$ and $30,000 \mu \mathrm{m}^{2}$. These values are marked by the large tick marks on the horizontal axis in panel (a).

the distribution of $N_{\text {spikes }}$ that enters into the calculation of the average in Eq. (8) is bell-shaped [see Fig. 1(b)], and the corresponding error bar shown in Fig. 1(a) is small.

In sharp contrast, Fig. 2(b) shows that very different behavior occurs for $A=30000 \mu \mathrm{m}^{2}$. This corresponds to the right side of the ISR curve where the noise is smaller. In this case, most trajectories exhibit either spiking or resting for the duration of the trace. Only two traces show a transition from spiking to resting, and no traces show transitions from resting to spiking. Furthermore, there are many trials for which $N_{\text {spikes }}=0$. Consequently, the distribution of $N_{\text {spikes }}$ is far from Gaussian [see Fig. 1(c)], and the corresponding error bar shown in Fig. 1(a) is misleadingly large. This is true for all the large error bars in Fig. 1(a). Subsequent figures will not include error bars for this reason.

The switching behavior described above suggests that attractors corresponding to resting and spiking behavior coexist, as has been noted in earlier publications. Figure 3 shows a (a)

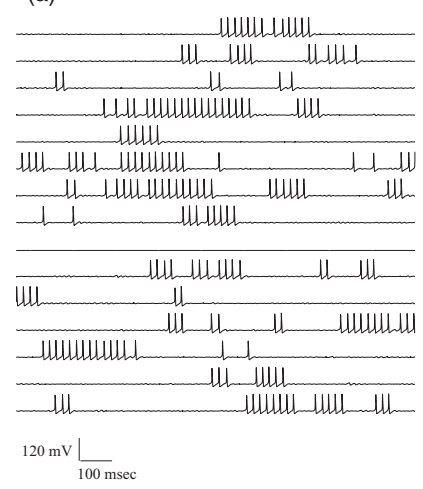

(b)

mumumememumumumumumumu

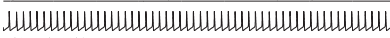

cumeum

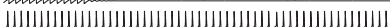

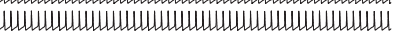

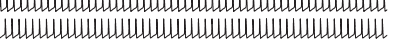

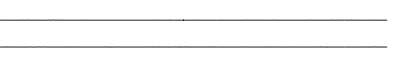

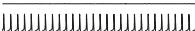

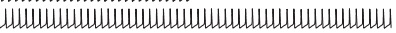

$\left.120 \mathrm{mV}\right|_{\overline{100} \mathrm{msec}}$
FIG. 2. Sample voltage traces at $I_{0}=6.8 \mu \mathrm{A} / \mathrm{cm}^{2}$ with membrane area (a) $750 \mu \mathrm{m}^{2}$ and (b) $30000 \mu \mathrm{m}^{2}$ (see the tick marks on the horizontal axis in Fig. 1). The traces were obtained from separate trials with randomly chosen initial conditions as described in the text. (a)

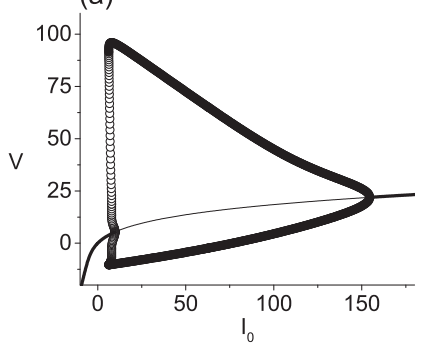

(b)

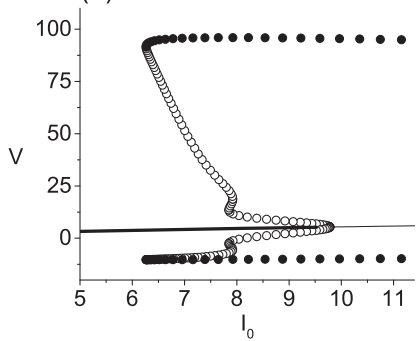

FIG. 3. Bifurcation diagram of the deterministic Hodgkin-Huxley neuron. Heavy (light) solid lines represent stable (unstable) equilibria. Solid (open) circles represent the asymptotic minimum and maximum values of the voltage during stable (unstable) spiking behavior. (b) A magnification of (a), revealing the multistable region.

bifurcation diagram that confirms this. In Fig. 3(a), the asymptotic behavior of the noise-free neuron's voltage is plotted versus the input current $I_{0}$, and Fig. 3(b) shows a magnification of the multistable region. For values of $I_{0}$ below $6.26 \mu \mathrm{A} / \mathrm{cm}^{2}$, the only attractor is a stable equilibrium. This corresponds to resting behavior. At $I_{0}=6.26 \mu \mathrm{A} / \mathrm{cm}^{2}$, stable and unstable limit cycles are created by saddle-node bifurcation. The stable limit cycle corresponds to spiking behavior. For increasing values of $I_{0}$, the stable limit cycle does not change very much in the range shown in Fig. 2(b). In contrast, the unstable limit cycle shrinks and eventually collapses onto the stable equilibrium in a subcritical Andronov-Hopf bifurcation at $I_{0}=9.78 \mu \mathrm{A} / \mathrm{cm}^{2}$. Between these two bifurcations, the stable resting equilibrium and the stable spiking limit cycle coexist.

Within this multistable region, noise of sufficient intensity can cause the switching behavior discussed above. In Fig. 4, we show the $n-V$ projections of the limit cycles and the equilibrium that are present for $I_{0}=6.8 \mu \mathrm{A} / \mathrm{cm}^{2}$, with magnifications in the vicinity of the equilibrium shown in the right panels. In addition, short segments of representative noisy trajectories are superimposed using the same projection, with $A=750 \mu \mathrm{m}^{2}$ (large noise) in the top panels, and $A=30000 \mu \mathrm{m}^{2}$ (small noise) in the bottom panels. These are the same parameters that were used in Fig. 2. Note that the projections of spiking behavior are traversed in a clockwise fashion. The corresponding voltage traces are shown as insets in the left panels for reference.

The top panels of Fig. 4 show that the large-noise trajectory switches back and forth between spiking and resting behavior. When spiking, the noisy trajectory approximately follows the deterministic stable limit cycle. When not spiking, the trajectory occupies a dispersed noisy cloud in the general vicinity of the equilibrium. The size of this cloud is on the order of the distance between the equilibrium and the limit cycle, and hence, transitions from resting to spiking behavior occur frequently. In contrast, the lower panels show a trajectory with much smaller noise. This trajectory begins in the spiking state, and it follows the stable limit cycle much more closely [compare Figs. 4(a) and 4(c)], until a noise fluctuation occurs of sufficient size and in just the right part of the cycle to kick the trajectory into the basin of the equilibrium [31]. It then spirals in toward the equilibrium, which is a focus, and hovers around the equilibrium. However, in this smaller-noise case, the noisy cloud that it occupies is very much smaller than the distance 

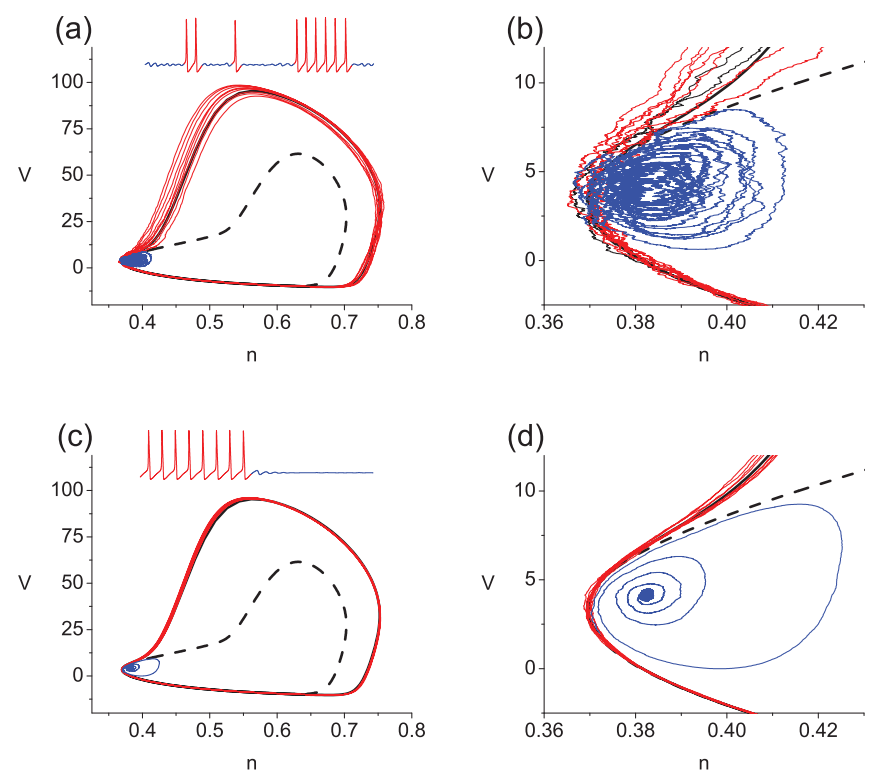

FIG. 4. (Color online) $n-V$ projections showing spiking/resting transitions for $A=750 \mu \mathrm{m}^{2}$ [panels (a) and (b); large noise] and $A=30,000 \mu \mathrm{m}^{2}$ [panels (c) and (d); small noise]. Insets in (a) and (c) show voltage traces of the same data, and panels (b) and (d) are magnifications of (a) and (c), respectively. In all cases, $I_{0}=$ $6.8 \mu \mathrm{A} / \mathrm{cm}^{2}$. Stable (solid) and unstable (dashed) limit cycles are shown in black; a stable resting equilibrium is also present. (a), (b) Relatively strong noise causes transitions back and forth between the two deterministically stable states. (c), (d) Here, the noise is relatively small. The spiking trajectory periodically approaches a critical region where noise might kick the state into the basin of the equilibrium. When it does so, the trajectory spirals into the equilibrium, which is too far from the basin boundary for the small noise to reignite spiking.

between the equilibrium and the limit cycle. Thus, it remains there, essentially forever: noise fluctuations of sufficient size to kick the trajectory back into the spiking state are extremely unlikely to occur in this case.

Transitions from spiking to resting are most likely to occur where the stable and unstable limit cycles are very close, and where both are close to the equilibrium. Note that in the spiking state, the trajectory repeatedly visits this critical region, which is near the "nose" on the left in Fig. 4. Thus, it has multiple opportunities to cross the basin boundary and fall into the equilibrium, even for very small noise. In contrast, for a transition from resting to spiking to occur, noise alone must cause a fluctuation of sufficient size to overcome the separation between the equilibrium and basin boundary. As noise decreases, this becomes less and less likely.

Accordingly, we hypothesize that depending on the initial condition and for sufficiently small noise intensity, trajectories either (1) approach the equilibrium and remain there, effectively forever, or (2) approach the spiking limit cycle and track it for a finite time before noise causes it to transition to the equilibrium, where it subsequently remains, effectively forever. This hypothesis suggests that if the initial transient time $T$ is chosen to be longer, then more trajectories that were tracking the limit cycle should undergo the transition to the resting state and then stay there. Consequently, the average firing rate (which is calculated after the transient time $T$ has

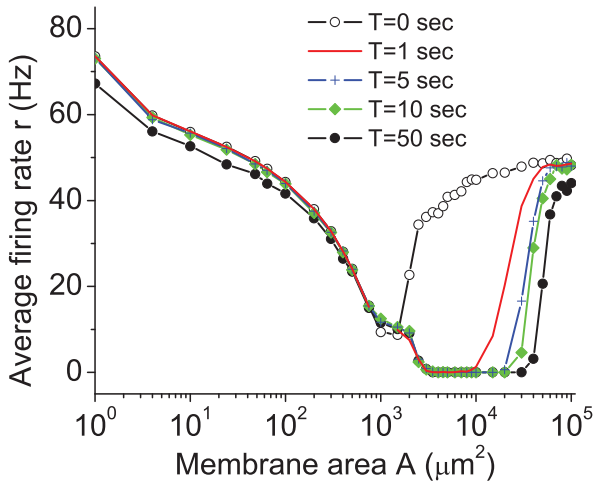

FIG. 5. (Color online) ISR curves obtained after discarding initial transients of length $T$ seconds, for $A=30000 \mu \mathrm{m}^{2}, I_{0}=$ $6.8 \mu \mathrm{A} / \mathrm{cm}^{2}, \tau=5 \mathrm{~s}, N=1000$, and various value of $T$. Initial conditions are chosen randomly. For small noise, trajectories starting in the basin of the spiking limit cycle spike for long times before being kicked to the resting state. By discarding longer transients, more such trajectories fall into quiescence, hence lowering the average firing rate.

passed) should decrease as the transient time $T$ is increased, at least in the small noise (large membrane area) regime.

Figure 5 shows the results of performing this test. ISR curves similar to that in Fig. 1 were calculated for various values of the transient time $T$, ranging from 0 to $50 \mathrm{~s}$. It is evident that for membrane areas larger than $1000 \mu \mathrm{m}^{2}$, the average firing rates are indeed smaller for larger values of $T$. In fact, four of the five curves fall to zero $\mathrm{Hz}$, and the range of membrane area over which this is seen increases with longer transient times $T$.

However, as these curves approach the right side of the figure, they rise back up to moderate firing rates. This seems surprising because the right side represents the lowest values of noise that we tested. This behavior can be understood by noting that for decreasing noise intensities, the probability for a spiking trajectory to transition to the resting equilibrium decreases. Thus, for lower noise intensities, spiking trajectories tend to spike for longer times. In the case of the extreme right side of Fig. 5, the noise is so small that spiking trajectories typically continue to spike for times in excess of the sum of the transient and measurement times, $T+\tau$, that we tested.

The protocol that we used to calculate the average firing rates was based on that used in Ref. [16], and it suggests that the average firing rates for the zero noise limit can be predicted. In particular, the initial conditions were selected randomly based on a uniform distribution within a particular region of state space. Let $P_{\text {spiking }}$ be the probability of randomly selecting an initial condition from the state space sample volume that is in the basin of attraction of the spiking limit cycle, and let $P_{\text {equilibrium }}$ be similarly defined, so that $P_{\text {spiking }}+P_{\text {equilibrium }}=1$. Then, since trajectories that asymptote to the resting equilibrium do not contribute to the rate calculated in Eq. (8), the predicted firing rate is simply $r_{\text {predicted }}\left(I_{0}\right)=P_{\text {spiking }} R$, where $R$ is the deterministic firing rate corresponding to the noise-free spiking limit cycle that is present with input current $I_{0}$. This is easily measured. 

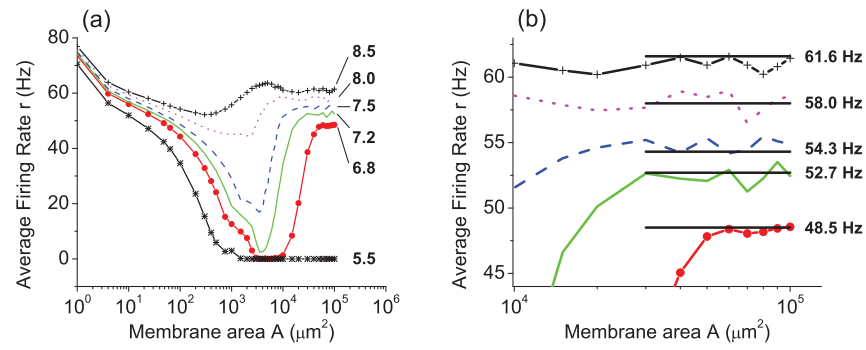

FIG. 6. (Color online) (a) ISR curves for different input currents $I_{0}$ as indicated by the numbers on the right (in $\left.\mu \mathrm{A} / \mathrm{cm}^{2}\right) \cdot \tau=5 \mathrm{~s}$, and $N=1000$. In the low noise limit, the curves approach asymptotic values of average firing rate that can be predicted. (b) Enlargement of the right side of (a), showing the approach to the predicted asymptotic average firing rates, which are indicated by the labeled black lines.

Because initial conditions are randomly chosen uniformly in the state space sample volume, we can estimate $P_{\text {spiking }}$ by measuring the fraction of the state space sample volume that is in the basin of the spiking limit cycle. That is, $P_{\text {spiking }}=$ $V_{\text {spiking }} / V_{\text {total }}$, where $V_{\text {total }}$ is the volume of the entire state space sample volume, and $V_{\text {spiking }}$ is the volume of only the portion that is within the basin of the spiking limit cycle. To do this, we selected an ensemble of initial conditions using a regularly spaced four-dimensional grid that spanned the entire state space sample volume. The membrane voltage variable $V$ was sampled every $10 \mathrm{mV}$, and the gating variables were each sampled every 0.01 , for a total of $n_{\text {total }}=10^{7}$ initial conditions. Each such initial condition was then integrated using our model with no noise for a time sufficient to determine if it ultimately approached the limit cycle or not. The number $n_{\text {spiking }}$ of such initial conditions that were attracted to the spiking limit cycle, divided by $n_{\text {total }}$, is then an estimate of $P_{\text {spiking }}$, and the predicted firing rate is

$$
r_{\text {predicted }}\left(I_{0}\right) \approx\left(\frac{n_{\text {spiking }}}{n_{\text {total }}}\right) R \text {. }
$$

This analysis is confirmed in Fig. 6, which shows several ISR curves calculated as described above for different values of the input current $I_{0}$. Note that the input currents are all from the bistable region shown in Fig. 3(b), except $I_{0}=$ $5.5 \mu \mathrm{A} / \mathrm{cm}^{2}$, for which no ISR effect is seen. For decreasing noise (i.e., increasing membrane area), the curves approach a plateau. This is magnified in Fig. 6(b), which also shows lines corresponding to the predicted deterministic firing rates of Eq. (9). The measured firing rates are in good agreement with the predicted rates for membrane areas larger than approximately $50000 \mu \mathrm{m}^{2}$.

\section{DISCUSSION}

In this work, we build on the results reported in Refs. [15,16] by describing the underlying dynamical structure and exploring its consequences. The ISR phenomenon was originally described using additive noise applied to either the voltage equation directly or to an excitatory synaptic term in the Hodgkin-Huxley model neuron. We began the current investigation by demonstrating that the ISR phenomenon also occurs when ion channel noise - the noise that occurs due to a finite number of stochastic ion channels-is implemented using the algorithm of Fox [27]. (To our knowledge, this is the first such demonstration, thus answering a question that was raised in Ref. [32].) Recently, it has been shown that the Fox algorithm does not capture certain aspects of real channel noise, and more realistic algorithms based on elaborate Markov processes have been proposed [33-35]. We confirmed that ISR also occurs with channel noise implemented as described in Ref. [33]. We do not report these results in detail, however, because we found that the ISR phenomenon can be explained in terms of the system's dynamical structure. Thus, we expect that ISR behavior would occur using any of these more realistic models of channel noise, or indeed, with any reasonable kind of noise.

The key insights are that, in the parameter region where the ISR effect occurs (and for the deterministic system), (1) the spiking limit cycle coexists with the stable resting equilibrium, (2) the basin boundary is relatively close to the stable limit cycle, and (3) the equilibrium is relatively far from the basin boundary. This deterministic structure gives rise, in the presence of small noise, to a significant asymmetry in the probabilities of transitions occurring from spiking to resting, and vice versa. As we described above, when this structure is present and there is small noise, it is much more likely to observe a transition from spiking to rest, than the reverse. A trajectory that begins by exhibiting spikes repeatedly visits a critical region near the basin boundary where a small noise fluctuation can cause it to be trapped, effectively forever, by the resting equilibrium. For example, see the small noise sample traces of Fig. 2(b) and the phase-space projections of Figs. 4(c) and 4(d). This explains the ISR phenomenon.

The presence of the deterministic dynamical structure described in the previous paragraph is dictated by the input current $I_{0}$. Figure 3 shows that this multistable structure only occurs in the small interval from (approximately) 6.26 to $9.78 \mu \mathrm{A} / \mathrm{cm}^{2}$. Thus, the ISR effect can only occur if $I_{0}$ is within this range and if small noise is present. In Ref. [36], the ISR phenomenon was investigated in a spatially extended Hodgkin-Huxley model neuron modeling a soma and axon. In that study, the input current and the current noise were applied independently and in various spatial locations, sometimes overlapping and sometimes not overlapping. It was noted that spikes elicited by an input current applied to the somatic region (i.e., one end of the cable) could generally propagate along the axon. However, if small noise coincided spatially with an appropriately tuned input current, spike propagation would fail, yielding the ISR effect. Furthermore, the effect was not seen when the noise did not coincide spatially with the input current. This is consistent with our view that the ISR effect requires both the multistable dynamical structure described above and small noise.

We find it important to emphasize that the quantitative aspects of the ISR phenomenon depend strongly on the details of how it is measured. This was noted in earlier publications, but the consequences and the connection to the dynamical structure was not made explicit. In particular, the choice of initial conditions and observation times are crucial, as we have examined and explained here.

In some ISR investigations [15,16], trajectories were obtained for analysis by using a single fixed initial condition. Here, we showed in Fig. 5 that the choice of transient time- the 
amount of time that the system is allowed to evolve from the initial condition before spikes are counted-dramatically affects the shape of the ISR curve. The reason for this is simply that in the parameter region of interest, the longer a noisy trajectory spends in the vicinity of the spiking limit cycle, the more likely it is to transition to the resting state, where it is very likely to remain forever if the noise is small. This effect is particularly relevant to the study in Ref. [32], in which many trajectories were constructed as follows: the first evolved from a single fixed initial condition in the basin of the spiking limit cycle, and each subsequent trajectory began from where the previous trajectory ended, thus obtaining essentially one extremely long trajectory (500 s) for analysis. Then, 50 such long trajectories were obtained. In the parameter region of interest, these very long trajectories are likely to be trapped at the equilibrium relatively early in their time evolution because of the transition probability asymmetry described above. Consequently, statistics calculated using such data are dominated by the resting state, and relatively few spikes are observed, yielding the ISR effect.

In other ISR investigations $[15,16]$, sets of randomly chosen initial conditions drawn from a specified region of the state space were used. Here, we showed (Fig. 6) that the smallnoise limiting behavior of the ISR curve can be predicted from knowledge of how the state space sample volume intersects the basins of the spiking limit cycle and the equilibrium. If one draws initial conditions from a different region of the state space, or if the random selection is not done uniformly, the quantitative details of the ISR curve will change. We note in particular that in order to observe the ISR effect, at least some initial conditions must be drawn from the basin of the spiking limit cycle in order to yield nonzero spike counts in the limit of zero noise. Without these, the ISR effect does not occur. This is illustrated by the $I=5.5 \mu \mathrm{A} / \mathrm{cm}^{2}$ curve shown in Fig. 6, since for this value of the input current, the limit cycle does not exist.

Finally, we note that the multistable structure that is required for the ISR phenomenon is not particularly unusual, and therefore the effect should be observable in a wide range of applications both inside and outside of neuroscience. For the Hodgkin-Huxley model studied here, the current interval in which multistability is present is quite small [compare Figs. 3(a) and 3(b)]. However, we suspect that similarly structured multistable regions occur more robustly in more biophysically realistic neurons that take into account, for example, a more complete complement of ion channels [37], neural modulators [38,39], and/or local environmental factors such as dynamic ion concentrations [40-42].

\section{ACKNOWLEDGMENTS}

M.U. acknowledges support from Grant No. 13643-49840 from the Turkish Council of Higher Education (YÖK) and the hospitality of the Krasnow Institute for Advanced Study at George Mason University. Publication of this article was funded in part by the George Mason University Libraries Open Access Publishing Fund and Bulent Ecevit University.
[1] A. A. Faisal, L. P. J. Selen, and D. M. Wolpert, Nature Rev. Neurosci. 9, 292 (2008).

[2] W. R. Softky and C. Koch, J. Neurosci. 13, 334 (1993).

[3] M. N. Shadlen and W. T. Newsome, J. Neurosci. 18, 3870 (1998).

[4] M. Häusser, I. M. Raman, T. Otis, S. L. Smith, A. Nelson, S. du Lac, Y. Loewenstein, S. Mahon, C. Pennartz, I. Cohen, and Y. Yarom, J. Neurosci. 24, 9215 (2004).

[5] E. Schneidman, B. Freedman, and I. Segev, Neural Comput. 10, 1679 (1998).

[6] J. J. Collins, C. C. Chow, and T. T. Imhoff, Nature (London) 376, 236 (1995).

[7] P. C. Gailey, A. Neiman, J. J. Collins, and F. Moss, Phys. Rev. Lett. 79, 4701 (1997).

[8] B. Kosko and S. Mitaim, Neural Networks 16, 755 (2003).

[9] M. Kawaguchi, H. Mino, and D. M. Durand, IEEE Trans. Biomed. Eng. 58, 1950 (2011).

[10] A. B. Neiman, T. A. Yakusheva, and D. F. Russell, J. Neurophysiol. 98, 2795 (2007).

[11] C. Li, L. Chen, and K. Aihara, PLoS Comput. Biol. 2, e103 (2006).

[12] A. S. Pikovsky and J. Kurths, Phys. Rev. Lett. 78, 775 (1997).

[13] J. J. Collins, T. T. Imhoff, and P. Grigg, Nature (London) 383, 770 (1996).

[14] D. Paydarfar, D. B. Forger, and J. R. Clay, J. Neurophysiol. 96, 3338 (2006).

[15] B. S. Gutkin, J. Jost, and H. C. Tuckwell, Naturwissenschaften 96, 1091 (2009).
[16] H. C. Tuckwell, J. Jost, and B. S. Gutkin, Phys. Rev. E 80, 031907 (2009).

[17] B. S. Gutkin, J. Jost, and H. C. Tuckwell, Europhys. Lett. 81, 20005 (2008).

[18] D. Guo, Cognitive Neurodynamics 5, 293 (2011).

[19] H. C. Tuckwell and J. Jost, J. Comput. Neurosci. 30, 361 (2011).

[20] M. Uzuntarla, Phys. Lett. A 377, 2585 (2013).

[21] E. Skaugen and L. Walloe, Acta Physiol Scand 107, 343 (1979).

[22] A. F. Strassberg and L. J. DeFelice, Neural Comput. 5, 843 (1993).

[23] J. T. Rubinstein, Biophys. J. 68, 779 (1995).

[24] C. C. Chow and J. A. White, Biophys. J. 71, 3013 (1996).

[25] J. A. White, J. T. Rubinstein, and A. R. Kay, Trends Neurosci. 23, 131 (2000).

[26] A. L. Hodgkin and A. F. Huxley, J. Physiol. 117, 500 (1952).

[27] R. F. Fox, Biophys. J. 72, 2068 (1997).

[28] G. Schmid, I. Goychuk, P. Hänggi, S. Zeng, and P. Jung, Fluct. Noise Lett. 4, L33 (2004).

[29] M. Ozer, M. Uzuntarla, M. Perc, and L. J. Graham, J. Theor. Biol. 261, 83 (2009).

[30] G. Schmid, I. Goychuk, and P. Hänggi, Europhys. Lett. 56, 22 (2001).

[31] The boundary between the basins of attraction of the spiking limit cycle and the resting equilibrium is the closure of the stable manifold of the unstable limit cycle. 
[32] H. C. Tuckwell and J. Jost, Physica A 391, 5311 (2012).

[33] D. Linaro, M. Storace, and M. Giugliano, PLoS Comput. Biol. 7, e1001102 (2011).

[34] J. H. Goldwyn and E. Shea-Brown, PLoS Comput. Biol. 7, e1002247 (2011).

[35] J. H. Goldwyn, N. S. Imennov, M. Famulare, and E. Shea-Brown, Phys. Rev. E 83, 041908 (2011).

[36] H. C. Tuckwell and J. Jost, PLoS Comput. Biol. 6, e1000794 (2010).
[37] B. Hille, Ion Channels of Excitable Membranes, 3rd ed. (Sinauer Associates, Inc., Sunderland, MA, 2001).

[38] A. Dovzhenok and A. Kuznetsov, PLoS One 7, e42811 (2012).

[39] P. Dayan, Neuron 76, 240 (2012).

[40] J. R. Cressman, G. Ullah, J. Ziburkus, S. J. Schiff, and E. Barreto, J. Comput. Neurosci. 26, 159 (2009).

[41] J. R. Cressman, G. Ullah, J. Ziburkus, S. J. Schiff, and E. Barreto, J. Comput. Neurosci. 30, 781 (2011).

[42] E. Barreto and J. R. Cressman, J. Biol. Phys. 37, 361 (2011). 